\title{
Association between [18F]-fluoro-2-deoxyglucose uptake and expressions of hypoxia-induced factor $1 a$ and glucose transporter 1 in non-small cell lung cancer
}

Takaoki Furukawa1* ${ }^{1 *}$ Yoshihiro Miyata' ${ }^{1}$ Kei Kushitani², Takahiro Mimae1, Yasuhiro Tsutani', Yukio Takeshima², Morihito Okada ${ }^{1}$

From World Society of Cardiothoracic Surgeons 25th Anniversary Congress, Edinburgh Edinburgh, UK. 19-22 September 2015

\section{Background/Introduction}

High maximal standardized uptake values (SUVmax) on [18F]-fluoro-2-deoxyglucose positron emission tomography (FDG-PET) are associated with inferior survival in non-small cell lung cancer (NSCLC).

\section{Aims/Objectives}

Here, we investigated the biological mechanisms underlying FDG uptake in NSCLC.

\section{Method}

This study included 133 patients with NSCLC (109 with adenocarcinoma and 24 with squamous cell carcinoma). The patients underwent tumor resection, at the latest, 4 weeks after FDG-PET. The SUVmax values for primary lesions were calculated based on FDG uptake. The expression of hypoxia-inducible factor $1 \alpha$ (HIF1 $\alpha)$ and glucose transporter 1 (GLUT1) was evaluated on immunostained tumor sections using six-point grading scales.

\section{Results}

SUVmax and the expression of HIF1 $\alpha$ and GLUT1 were significantly higher in squamous cell carcinoma than in adenocarcinoma ( $<<0.001, \mathrm{p}=0.034$, and $\mathrm{p}<0.001$, respectively). In adenocarcinoma, but not squamous cell carcinoma, SUVmax, HIF1 $\alpha$, and GLUT1 correlated with various clinicopathological factors relating to malignancy, and SUVmax and GLUT1 were associated with disease-free survival (DFS) $(\mathrm{p}<0.001$ and $\mathrm{p}=$ $0.029)$ and overall survival (OS) $(\mathrm{p}<0.001$ and $\mathrm{p}=$ 0.033 , respectively). Moreover in adenocarcinoma, HIF1 $\alpha$ and GLUT1, GLUT1 and SUVmax, and HIF1 $\alpha$ and SUVmax were significantly correlated $(\mathrm{p}<0.001$ for all), suggesting that HIF1 $\alpha$-induced GLUT1 might influence SUVmax values on FDG-PET.

\section{Discussion/Conclusion}

In lung adenocarcinoma, but not squamous cell carcinoma, HIF1 $\alpha$, and GLUT1 expressions indicate tumor aggressiveness pathologically, and might explain high FDG uptake on PET and correlate with poor prognosis.

\section{Authors' details}

${ }^{1}$ Department of Surgical Oncology, Research Institute for Radiation Biology and Medicine, Hiroshima University, Hiroshima, Japan. ²Department of Pathology, Graduate School of Biomedical Sciences, Hiroshima University, Hiroshima, Japan.

Published: 16 December 2015

\section{doi:10.1186/1749-8090-10-S1-A211}

Cite this article as: Furukawa et al:: Association between [18F]-fluoro-2deoxyglucose uptake and expressions of hypoxia-induced factor 1a and glucose transporter 1 in non-small cell lung cancer. Journal of Cardiothoracic Surgery 2015 10(Suppl 1):A211. 